Review

\title{
Edelman's view on the discovery of antibodies
}

\author{
Domenico Ribatti ${ }^{\mathrm{a}, \mathrm{b}, *}$ \\ a Department of Basic Medical Sciences, Neurosciences and Sensory Organs, University of Bari Medical School, Bari, Italy \\ b National Cancer Institute "Giovanni Paolo II", Bari, Italy
}

\section{A R T I C L E I N F O}

\section{Article history:}

Received 23 January 2015

Received in revised form 8 February 2015

Accepted 15 February 2015

Available online 21 February 2015

\section{Keywords:}

Antibody

Clonal selection theory

Immune globulins

History of medicine.

\begin{abstract}
A B S T R A C T
Gerald M. Edelman began working to the structure of antibodies when joined as graduate student the laboratory of Henry Kunkel in 1958 at the "Rockefeller University" in New York, obtaining his doctorate in 1960. Edelman's focus on the structure of antibodies led to the 1972 Nobel Prize in Physiology or Medicine along with Rodney R. Porter. Edelman and Porter decided to approach the problem of antibodies structure by splitting. In 1959, Porter published a report in which he used the enzyme papain to cleave the antibody molecule into three pieces of about 50,000 Da, corresponding to the two Fab (antigenbinding) and constant Fc (crystallizable) fragments. In the same year, Edelman showed that reduction of the disulfide bonds of antibodies in the presence of denaturizing agents led to dissociation of the molecule into smaller pieces, now known to be the light $(\mathrm{L})$ and heavy $(\mathrm{H})$ chains.
\end{abstract}

(c) 2015 European Federation of Immunological Societies. Published by Elsevier B.V. All rights reserved.

\section{Biographic note}

Gerald M. Edelman (Fig. 1) died on 17 May 2014, aged 84 years, in La Jolla, California. Born in New York City, on July 1, 1929, he studied medicine at the University of Pennsylvania, where he received medical degree in 1954.

From 1955 to 1957, Edelman served in the Army Medical Corps in Paris, after which began working to the structure of antibodies, when joined as graduate student the laboratory of Henry Kunkel in 1958 at the "Rockefeller University" in New York, obtaining his doctorate in 1960. As Edelman pointed out: "In 1958, I was fascinated by the specificity of antigen recognition by antibodies. At the same time, I was frustrated as a chemist by the heterogeneity of the $\gamma$-globulin fractions containing these antibodies. Free boundary electrophoresis by Arne Tiselius and Elvin Kabat revealed a stark contrast between the distribution of net charge of these proteins as compared with that of other serum proteins. I was driven by the hope of resolving this heterogeneity and had the dream, naive as it was at the time, that by doing the primary structure of antibody molecules, the basis of their specificity would be revealed." [1].

Edelman's focus on the structure of antibodies led to the 1972 Nobel Prize in Physiology or Medicine along with Rodney R. Porter

\footnotetext{
* Corresponding author at: Department of Basic Medical Sciences, Neurosciences and Sensory Organs, University of Bari Medical School, Policlinico-Piazza G. Cesare, 11, 70124 Bari, Italy. Tel.: +39080 5478326; fax: +390805478310.

E-mail address: domenico.ribatti@uniba.it
}

(1917-1986) (Fig. 2) "for their discoveries concerning the chemical structure of antibodies". Their advance "incited a fervent research activity the whole world over, in all fields of immunological science, yielding results of practical value for clinical diagnostics and therapy," the Nobel committee said. The award recognized Edelman's work on the descriptions of the heavy $(\mathrm{H})$ and light $(\mathrm{L})$ polypeptide chains, and Porter's on the distinct binding domain (antigen-binding or Fab fragment) of antibodies.

Gerald Edelman's career has been eclectic, and highly successful. After the prize, Edelman's group expanded its interests in several areas, including carbohydrate-binding proteins on the surface membrane of cells, and tissue morphogenesis. He found a class of proteins, called cell adhesion molecules, thought to play an important role in embryonic development.

In the late 1970s, Edelman turned his attention to the brain and by 1991, he was director of the "Neuroscience Institute" at "Rockefeller University". In 1993, he moved the institute to the La Jolla neighborhood of San Diego. From 1995 the institute was part of the Scripps Research Institute campus; it moved to another location in La Jolla in 2012.

Edelman formulated a theory to explain the development and organization of higher brain functions in terms of a process known as neuronal group selection. This theory was presented in his 1987 volume Neural Darwinism. A new, biologically based theory of consciousness extending the theory of neuronal group selection is presented in his 1989 volume The Remembered Present. A subsequent book, Bright Air, Brilliant Fire, published in 1992, continues to explore the implications of neuronal group selection and neural evolution for a modern understanding of the mind and the brain. 


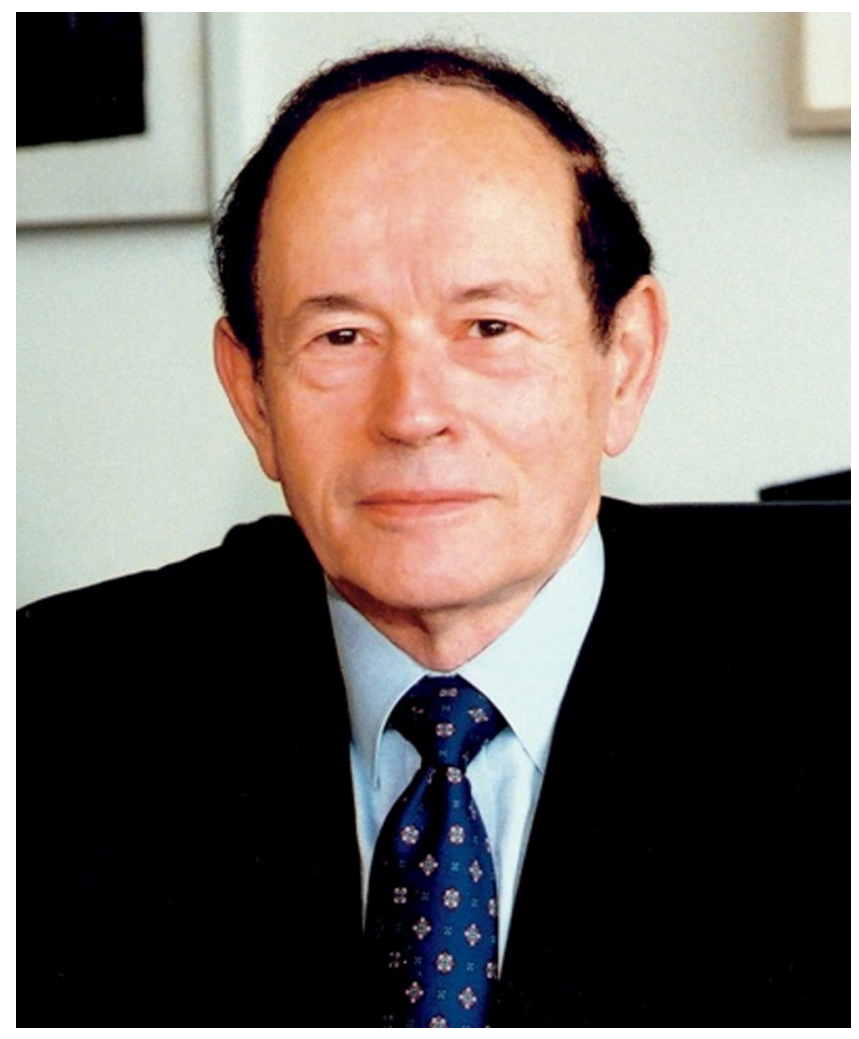

Fig. 1. A port trait of Gerald M. Edelman.

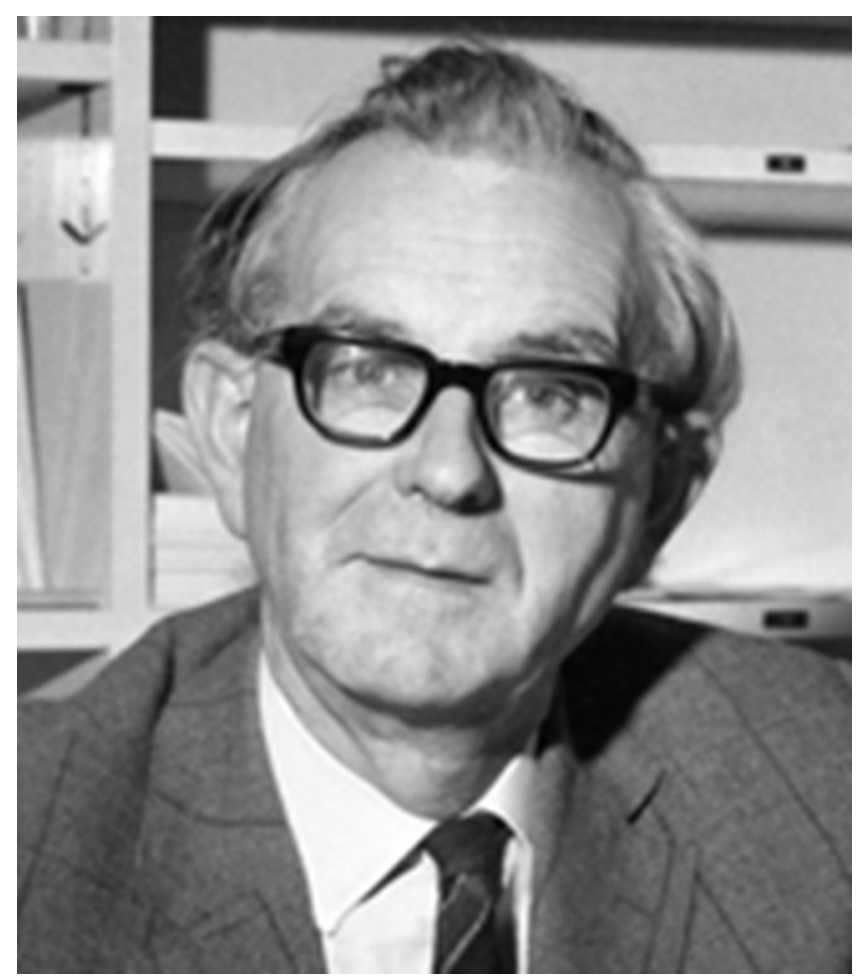

Fig. 2. A port trait of Rodney R. Porter.

\section{The discovery of the structure of immune globulins}

In 1950s, immunology was largely a sub-discipline of microbiology with a strong emphasis on serology and vaccines. The prevailing theories were instructional: accordingly, the antigen or hapten

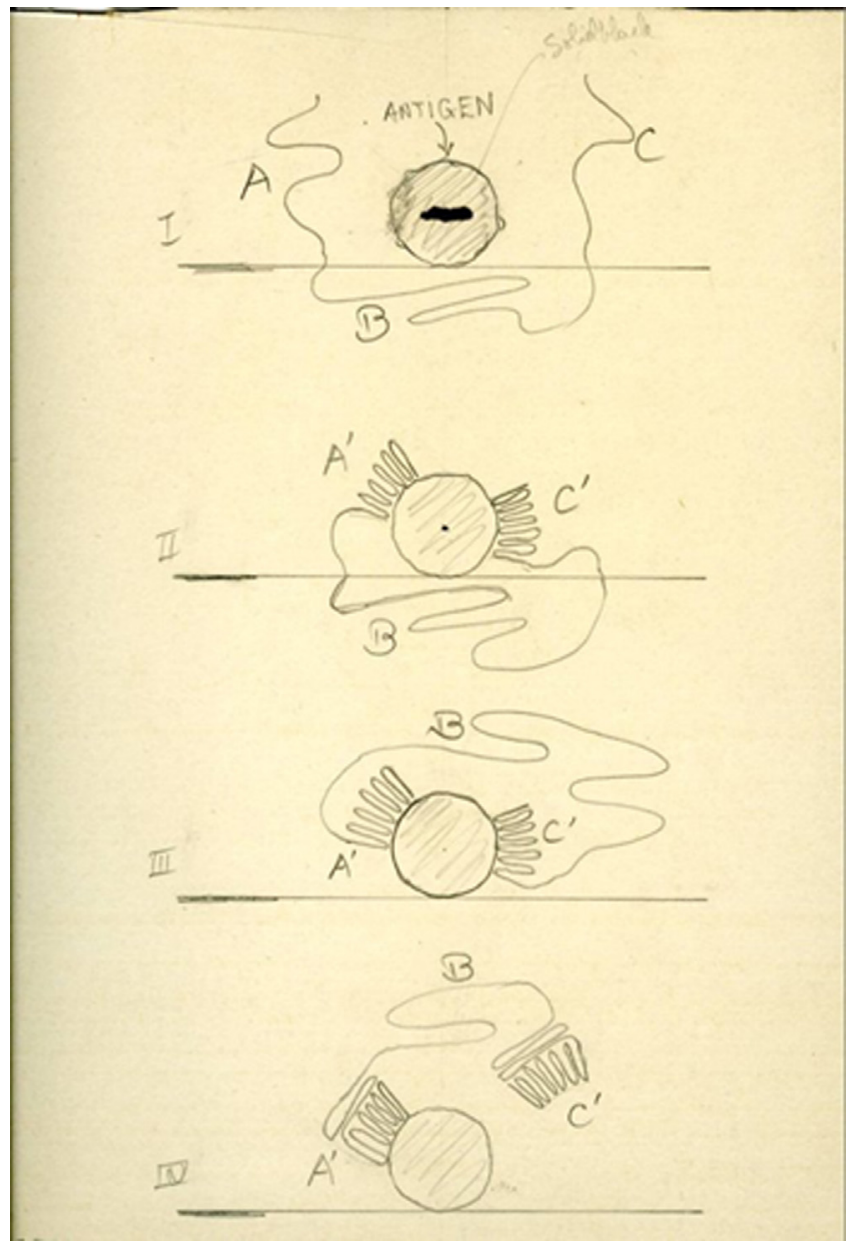

Fig. 3. Drawing of antigen and antibodies by Linus Pauling.

served as a template for the folding of the antibody-combining site [2] (Fig. 3). The "instructional" theory, as it was proposed by Pauling, arose from the universally accepted concept that the antibody repertoire had to be transcendental to protect the animal. As the number of genes had to be lower than the number of antigens that are recognized, the only solution seemed to be the "instructional" theory. Accordingly, the antibody was synthesized or folded, in specific ways in spatial contact with the antigen, which acted as a template.

In the opinion of Edelman: 'Pauling looked only at the chemical level. He ignored the fact that the body did not produce antibodies to its own antigens, a fact difficult to account for simply by instructional folding.' [1].

Within the period from 1959 to 1969, instructional theories were abandoned in favor of selection theories [3,4], that took their inspiration from Darwinian two-step variation and selection, leading to alterations in population structure. The various immune globulins were synthesized prior to antigen exposure and made up a repertoire of variant proteins.

In his paper entitled "The natural selection theory of antibody formation", Jerne wrote that: "Among the comparatively small number, perhaps a few thousand, of antigen-antibody systems investigated, cross-reactions are by no means rare, suggesting that the number of specific configurations which globulin molecule can exhibit is large but limited. Since normal mammalian serum contains more than $10^{17}$ globulin molecules per millimeter, these may include a million $10^{11}$ fractions of different specificity. This would seem an amply sufficient number.". 


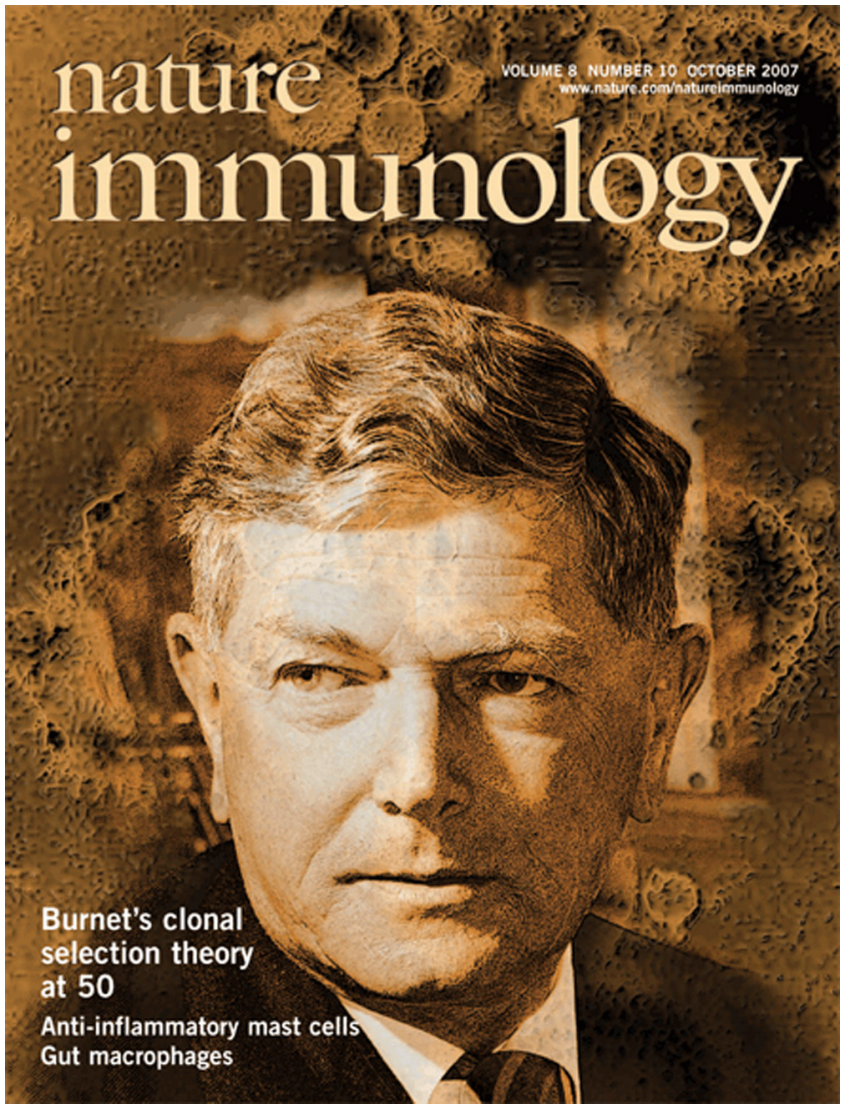

Fig. 4. The cover of "Nature Immunology" dedicated to Burnet's clonal selection theory.

Burnet suggested that an antigen might induce modifications of those enzymes involved in globulin synthesis so that a protein with the required specificity might be formed. This hypothesis concerned the manner in which the body normally failed to make antibodies to its own components, the "self-marker" concept, according to which the distinction between the 'self' and the 'notself' is based on a limited number of recognizable components in each cell, the combination of which constitute the specific 'self pattern' of the organism. The basis of clonal selection theory is that the specific capacity of a cell to react immunologically is conferred on by genetic processes and not by the intrusion of a pattern from the antigen. The clonal selection theory advanced the concept that antibodies were natural globulins that possessed an affinity for antigens and are selected from a large group of pre-existing globulins.

In the opinion of Edelman: "Burnet was insufficiently respectful of the biochemical rules, the syntax that would ultimately reveal in detail the origins of antibody diversity and specificity." [1].

Edelman and Porter decided to approach the problem of antibodies structure by splitting. Porter's experiments focused on a specific antibody from the gamma globulin fraction of rabbit serum called immunoglobulin G or IgG. In 1959, Porter published a report in which he used the enzyme papain to cleave the antibody molecule into three pieces of about 50,000 Da, corresponding to the two Fab (antigen-binding) and constant Fc (crystallizable) fragments [5] (Figs. 4 and 5). Papain hydrolyzed peptide bonds in IgG and produced three fragments (I, II, and III). The three fragments had similar molecular weights ( $50 \mathrm{kDa}$ ) but different charges.

The crystals of the Fc fragments coming from antibodies with different specificities were practically homogeneous. On the other hand, the lack of capacity of Fab fractions to form crystals

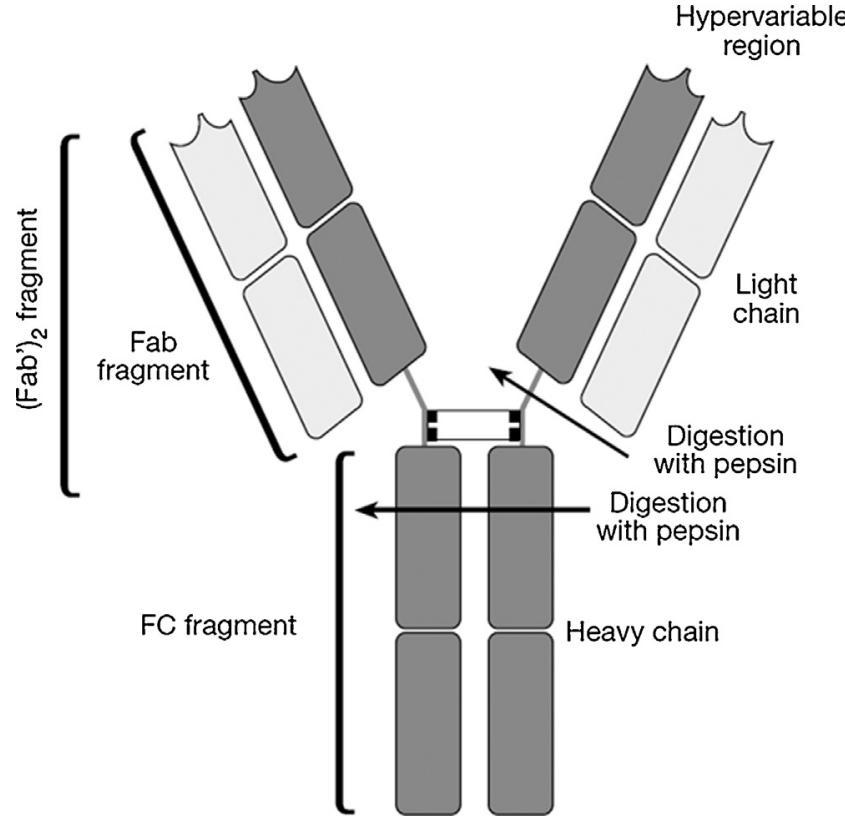

Fig. 5. Monomeric unit structure of immune globulins. Enzymatic digestion with papain produces two fragments that bind the antigen (Fab) and one that crystallizes (Fc). Treatment with pepsin produces one large fragment $\left(\mathrm{Fab}^{\prime}\right) 2$ and smaller ones (pepsin peptides).

correlated the antigenic specificity with the structural heterogeneity and differences in its amino acid sequence.

In the same year, Edelman showed that reduction of the disulfide bonds of antibodies in the presence of denaturizing agents led to dissociation of the molecule into smaller pieces, now known to be the light $(\mathrm{L})$ and heavy $(\mathrm{H})$ chains [6]. Because the molecular weight of the original IgG molecule is $150 \mathrm{kDa}$, he concluded that the IgG molecule consisted of two heavy and two light chains linked by disulfide bonds and noncovalent interactions. A Y-shaped configuration was proposed and then confirmed through electron microscopy and X-ray diffraction study. Thereafter, two antigenic types of light chains, denominated $\kappa$ and $\lambda$ chains were described (Fig. 6).

At that time, Sanger [7] had completed the sequencing of insulin and Stein and Moore [8] were sequencing ribonuclease.

As Edelman wrote: "I had the thought that if I broke the disulphide bonds in gamma globulin, I could look in the analytical centrifuge to see what happened to the molecule (...) It looked

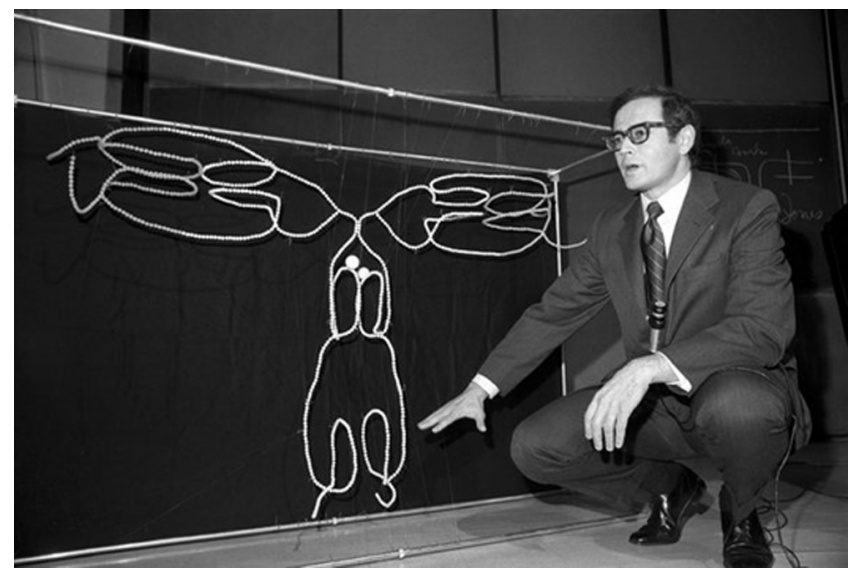

Fig. 6. Gerald M. Edelman at Rockefeller University in 1972, in front of a gamma globulin model. 
like the treated molecule hardly migrated at all compared to the original one (...) I come to the conclusion that the molecule must have composed of more than one polypeptide chain linked by disulphide bonds (...) My paper, by the way, was almost a phantom. It was a single page with one introductory sentence, a one sentence conclusion and an equation in the middle. The conclusion was that the gamma globulin molecule consists of multiple subunits of polypeptide chains." [9].

Following upon demonstration by Hilschmann and Craig [10] that light chains were composed of variable and constant regions, Edelman et al. [11] established the complete amino acid sequence of a myeloma protein. He picked one IgG myeloma protein that had obtained in over $200 \mathrm{~g}$-amounts from a patient who had been exchange-transfused because his myeloma protein was caused viscosity problems, and completed the sequence of the whole molecule [11].

In the meantime, Press and Hogg [12] had most of their heavy chain and comparison of the data from both laboratories with those on other heavy chains confirmed the hypothesis that there were $\mathrm{V}$ and $C$ regions from these chains.

Edelman [13] proposed the domain hypothesis predicting that the amino acid sequence in each region would fold into a compact domain with a single disulphide bond in its interior. However, this hypothesis proposed that each pair of domains across a symmetry axis ( $\mathrm{L}-\mathrm{H}$ or $\mathrm{H}-\mathrm{H})$ would carry out a distinct function and, finally, domains were presumed to have arisen by gene duplication of an ancestral gene coding for a single domain.

\section{The discovery of L-chain polypeptide in the urine of multiple myeloma patients}

The application of protein sequencing methodology to myeloma proteins during the 1960 s provided fundamental information about the structural nature of antibody diversity. Multiple myeloma patients produced large amounts of a single gamma globulin called myeloma protein that appeared to lack the heterogeneity of the molecules from normal individuals. They were each homogeneous and each differed from the others in the net charge. Myeloma proteins were invaluable in the resolution of antibody structure because they are produced in large quantities and are usually homogeneous in a patient, whereas in a normal immune response the antibodies are heterogeneous in composition.

Moreover, some of these patients excreted a smaller protein (Bence-Jones protein, first described in 1847 by Henry BenceJones) in their urine that was homogeneous and had unusual solubility properties during heat denaturation. When heated, the urine becomes cloudy in much the same fashion as urine containing albumen. However, on continued heating the urine becomes clear. Bence-Jones proteins were discovered to be monoclonal light chains quite independently of Edelman, and variable and constant regions, first seen in these Bence-Jones proteins, were crucial for a revolutionary change in the paradigm "One gene-one polypeptide"-again not by Edelman.

As Edelman wrote: "Given my hypothesis about myelomas, the thought arose that perhaps Bence-Jones protein was one of the chains of the myeloma protein that spilled into the urine because of its relatively low molecular weight (about 22,000)" [9]. Edelman decided to study these proteins and after he compared reduced myeloma proteins from a number of different patients, he demonstrated that each protein when reduced and alkylated and subjected to starch gel electrophoresis, had a unique migration pattern [14].

Bence-Jones proteins were simple excreted light chains. Edelman heated a sample of light chain obtained from normal human serum gamma globulins and demonstrated that they had the behavior of Bence-Jones proteins becoming insoluble and than resolubilizing with continued heating [15]. In 1967, Putman et al. demonstrated that different Bence-Jones proteins were different in their peptide sequence.

As Edelman wrote: "The key experiment was to use the recently discovered starch gel electrophoresis to take a whole bunch of patients' urine samples, separate their Bence-Jones proteins, and simultaneously separate and compare their serum proteins. When we ran the electrophoresis we demonstrated that the light chains were the Bence-Jones proteins and they were pure and no two had the same mobility pattern." [9].

Edelman showed that each antibody chain has a tandem series of repeating homology units roughly 110 amino acid residues in length called immunoglobulin domains, which fold independently into a compact globular structure. All proteins that exhibit this structural motif belong to the immunoglobulin gene super family.

Since the discovery of Edelman, it has been clear and progressively established that antibodies are multi-faceted proteins, capable of an extraordinary array of important functions. Not only major players in protection against invading pathogens, they play critical roles in inflammatory and autoimmune diseases, their induction is central to many vaccine strategies, and they are an unrivalled platform for engineering highly effective diagnostic and therapeutic reagents. Novel approaches to engineer effective therapeutic antibodies will also be a key theme, including strategies to control antibody half-life and bio-distribution.

\section{References}

[1] Edelman GM. Interview with Gerald M. Edelman. Part I. Bioessays 2004:26:204-13.

[2] Pauling L. Theory of structure and process of formation of antibodies. J Am Chem Soc 1940;62:2643-57.

[3] Jerne NK. The natural selection theory of antibody formation. Proc Natl Acad Sci USA 1955;41:849-57.

[4] Burnet FM. The clonal selection theory of acquired immunity. Nashville: Vanderbilt University Press; 1959.

[5] Porter RR. The hydrolysis of rabbit $\gamma$-globulin and antibodies with crystalline papain. Biochem J 1959;73:119-26

[6] Edelman GM. Dissociation of $\gamma$-globulin. J Am Chem Soc 1959;81:3155.

[7] Stretten A. The first sequence. Fred Sanger and insulin. Genetics 2002; $162: 527-32$

[8] Hirs CH, Moore S, Stein WH. The sequence of the amino acid residues in per formic acid-oxidized ribonuclease. J Biol Chem 1960;235:633-47.

[9] Edelman GM. Biochemistry and the sciences of recognition. J Biol Chem 2004;279:7361-9.

[10] Hilschmann N, Craig LC. Amino acid sequence studies with Bence Jones proteins. Proc Natl Acad Sci USA 1965;53:1403-9.

[11] Edelman GM, Cunningham BA, Gall WE, Gottlieb PD, Rutishauser U, et al. The covalent structure on entire $\gamma \mathrm{G}$-immunoglobulin molecule. Proc Natl Acad Sci USA 1969;63:78-85.

[12] Press EM, Hogg NM. Comparative study of two immunoglobulin G-Fdfragments. Nature 1969;223:808-10.

[13] Edelman GM. The covalent structure of a human $\gamma \mathrm{G}$-immunoglobulin. XI. Functional implications. Biochemistry 1970:9:3197-204.

[14] Edelman GM, Poulik MD. Studies on structural units of the globulins. J Exp Med 1961;113:861-84.

[15] Edelman GM, Gally JA. The nature of Bence Jones proteins. J Exp Med 1962;116:207-27. 